\title{
Hybrid structural layout of wing box for small aeroplane
}

\author{
Lada Zudova ${ }^{1, *}$, Victor Fomin ${ }^{2}$, Danil Fomin ${ }^{2}$ and Konstantin Zudov ${ }^{1}$ \\ ${ }^{1}$ Moscow Institute of Physics and Technology \\ ${ }^{2}$ Central Aerohydrodynamic Institute, Zhukovsky, Russia
}

\begin{abstract}
The algorithm of optimization of the hybrid structural layout for the wing box has been developed. The wing box consisted of two parts; one of them (heavily loaded) had hybrid (lattice-truss) layouts, the other had conventional (black metal) layout. These parts was connected by use of interface structure. The basic objectives were sizing of load-bearing elements for each of the layouts, and to search for the interface position, providing minimum weight of the wing box structure. These tasks were solved with use of genetic algorithm. At searching for the interface optimal position the results of parametric FEM calculations were additionally used. A rational variant (65-73\%) of the relative length of the wing box zone with lattice-truss structural layout has been obtained for the aircraft of regional airlines. This variant meets the constraints, and has the weight benefit (13$17 \%$ ) compared with conventional structural layout.
\end{abstract}

\section{Introduction}

For competitiveness preservation at satisfaction of new requirements on ecology, comfort and safety, at designing of perspective airframes there is a necessity of substantial reduction of the structure weight in comparison with conventional designs. The use of composite materials in airframes is lately the most widespread way to solve this problem.

Conventional structural layouts were formed and developed for isotropic (homogeneous) metal designs. It does not allow to raise considerably weight efficiency of the perspective airframes using composite materials in high-loaded parts of structures. For this reason recently the great attention is given to research new structural layouts, basing on use of the composite materials with taking into account not only of their advantages in specific strength, but also of their disadvantages inherent in modern composite materials at their use in loadbearing parts of the structure.

The most part of disadvantages is connected with considerable difference in stiffness characteristics of binding and reinforcing fibres, and also with insufficiently high properties of the modern binding. At service of a composite structure there can be a degradation of the characteristics of the composite package. It is connected with climatic influences (hot/wet). At the global stretching strain of the composite package of $0.3-0.4 \%$ the microcracks can already appear in binding of the monolayers located across loading, while the monolayers

\footnotetext{
${ }^{*}$ Corresponding author: zudova.lada@mail.ru
} 
located along this loading can maintain deformations to $1.5-1.7 \%$ without cracking. These microcracks at the further service can be transformed into delamination. It sharply reduces durability of the composite package at long service. Besides, unidirectional composite packages can collapse because of the certificated impact. It demands creation of special protection system for such structures.

The analysis of advantages and disadvantages of different layered packages has shown that for concrete maximum level of loads on an investigated design, it is possible to define the most suitable structural layout from the point of view of weight efficiency [1]. In the given work the algorithm of development of hybrid layout for composite wing box has been generated, and its validation by designing the wing box of the plane of small aircraft was carried out. For composite structures three kinds of structural layouts were considered: conventional, truss and lattice.

\section{Optimization algorithm}

The investigated wing box structure was divided onto two parts: high-loaded root section, and low-loaded end section (fig. 1). These parts were assembled with use of interface joints [2], which were developed for connection of sections with different structural layouts (lattice and conventional). Such joint was effective for the structures in which the longitudinal forces were dominating in external load.

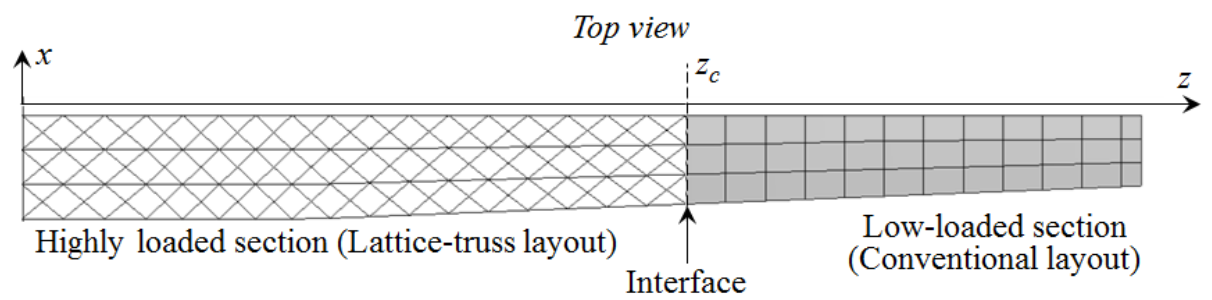

Fig. 1. Dividing of wing box onto two sections according to loading level

Two tasks were solved:

1) determination of optimum co-ordinate $z_{\mathrm{c}}$ of the interface between two sections with different structural layouts,

2) and determination of rational parameters of the wing box structure from the point of view of a minimum of weight, and with satisfaction of requirements (constraints) on strength and buckling.

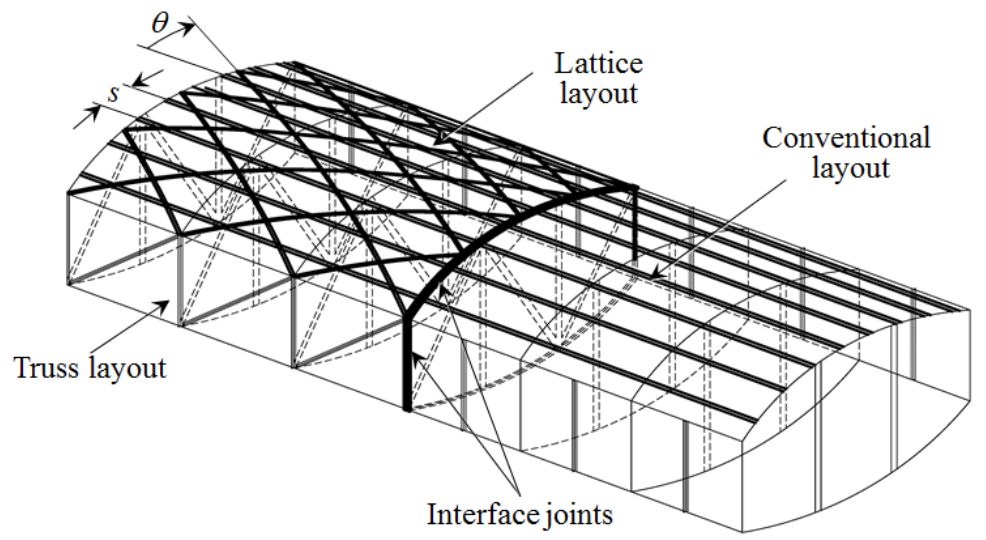

Fig. 2. Wing box sections with different structural layouts 
For the wing box three types of structural layout were used: conventional, truss and lattice. Before solving the tasks specified above, the analysis of behaviour of load-bearing elements of each of these layouts has been carried out at the levels of deformations which were characteristic for service of the structure. The types of structural layouts for root and end parts have been chosen basing on results of this analysis. The requirements on minimum stiffness were critical ("weight-making") for the end section, therefore conventional layout "black metal" has been chosen for this section. Parameters of high-loaded root section were defined mainly by conditions on strength and buckling. For this section, the hybrid layout containing lattice and truss parts has been chosen (fig. 2). In the truss part of the structure the hybrid rods were used [3].

For the skins of conventional layout quasi-isotropic packing of CFRP was used $\left[ \pm 45^{\circ} / 0^{\circ} / 90^{\circ}\right]$; cylindrical parts of hybrid rods of truss layout were formed with CFRP packing, close to unidirectional (a dominance of layers $\pm 15^{\circ}$ and $0^{\circ}$ ); and for lattice layout unidirectional packing of layers $\left[0^{\circ}\right]$ was considered.

The brief flow-chart of optimization algorithm is shown on fig. 3. The standard scheme of description of optimization problem is shown below.

Minimize:

$F\left(s, \theta, Z_{c}\right)=\sum_{i=1}^{i=2} W_{i}\left(s, \theta, Z_{c}\right) \rightarrow \min \quad\left(W_{1}, W_{2}-\right.$ weights of the lattice-truss and conventional parts of the wing box accordingly).

With respect to:

optimization variables $\left(s, \theta, Z_{c}\right)$ and geometry parameters of load-bearing elements.

Subject to:

constraints:

$\left\{\lambda_{s}, \lambda_{g b}, \lambda_{l b}\right\} \geq 1$ (reserve factors on strength, global and local buckling).

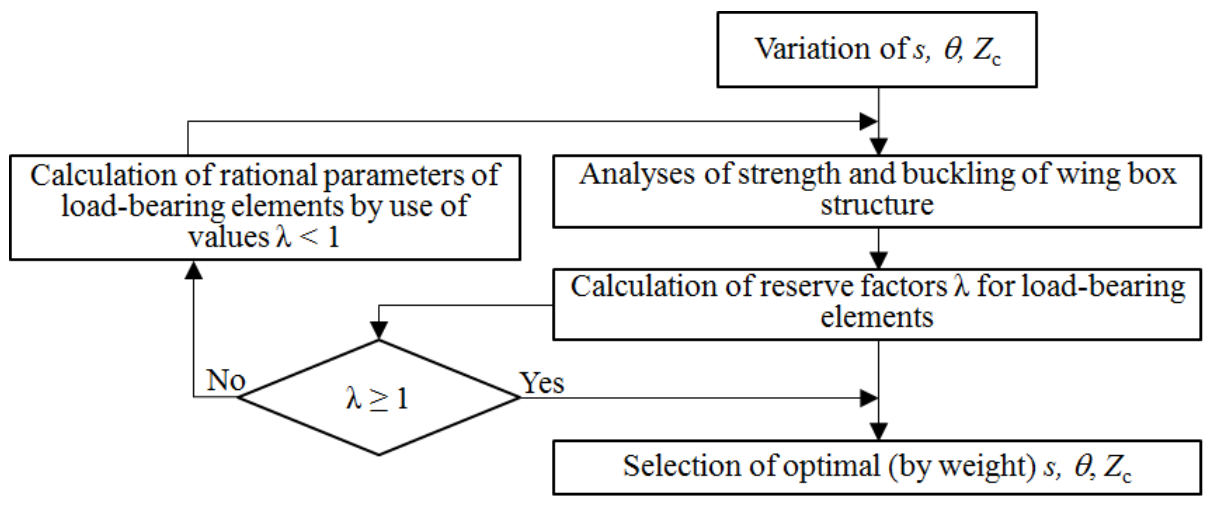

Fig. 3. Brief flow-chart of optimization algorithm

In frame of genetic optimization algorithm ([4, 5]) the points (individuals) were considered. Each of them consisted of three varied components: distance $z_{\mathrm{c}}$ from a fuselage to the interface joint of root and end sections, step $s$ of stringers, coinciding with distance between longitudinal lattice ribs, an angle $\theta$ between spiral ribs in the lattice part of the root section. A certain variant of the wing box structure corresponds to each of the points.

For each of the points obtained in the genetic process, the stress-strain state was calculated and the analyses of strength and local and global buckling with calculation of corresponding reserve factors $\lambda$ for the points were carried out. If for one or more load-bearing elements any of the requirements was not fulfilled $(\lambda<1)$, for the corresponding element (elements) new values of geometry parameters, which influence strength or buckling of the structure were calculated. For example, a width of the rib could be such parameter for a lattice rib, for 
skins - thickness of a composite package, for stringer - width or height of webs and flanges, etc. Further for this point the strength and buckling analyses repeated. At performance of conditions $\lambda \geq 1$ for all requirements on strength and buckling for current variant of the wing box structure this variant was selected into a group of rational solutions. The solution to which the minimum weight of the wing box structure corresponded was considered as optimal.

\section{Validation of algorithm}

Validation researches consisted in application of the optimization algorithm for the weight analysis of the wing box of regional plane. The strength and buckling analyses were carried out for three critical loading cases, which influenced results of the weight analysis in the greatest measure.

The most difficult operation of the algorithm was connected with providing the automated process of matching of different structural layouts in adjacent sections, without which it was inconvenient to provide rather light interface joints. This difficulty was caused by the fact that the varied parameters $z_{\mathrm{c}}$, s and $\theta$ were not completely independent variables. The value of the constant step s of stringers in the conventional structure layout was connected with step $b$ of structural cells of lattice grid; the value $b$, in turn, depended on the angle $\theta$ between spiral ribs. The position of the joints between root and end sections also was influenced by parameters of the structural cell.

In process of the validation researches the matching of different structural layouts in the adjacent sections was carried out in semi-automatic mode, with the analysis of results of parametrical calculations in which values of the parameters $z_{\mathrm{c}}, s$ and $\theta$ were varied in the ranges with discrete values. The example of results of parametrical calculation of the structure weight for determination of the most effective position of the interface joint between the root and end sections is shown in fig. 4.

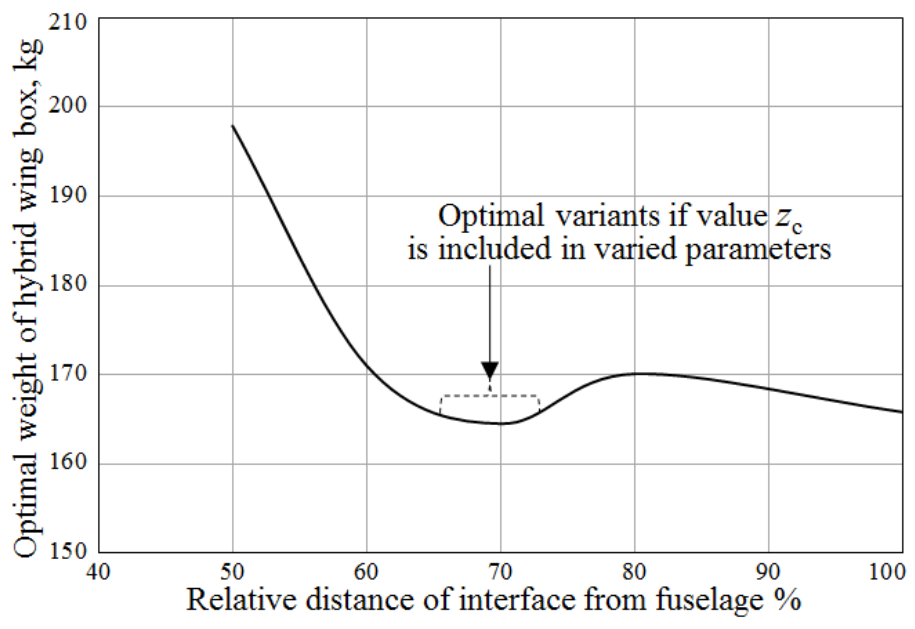

Fig. 4. Example of parametric calculation of wing box structure weight.

The range of solutions for $z_{\mathrm{c}}$, close to the optimal variant has been determined by results of this calculation. According to these solutions optimum value $z_{\mathrm{c}}$ were located in the range of $65-73 \%$ of length of the wing box structure. The weight of the structure for variants from this range turned out to be less (on 13-17\%), than an alternative variant with conventional structural layout. 


\section{Conclusions}

A hybrid structure of wing box consisting sections with different structural layouts has been proposed. The wing box consisted of two sections; one of them (heavily loaded) had hybrid (lattice-truss) layout, the other had conventional one (black metal). These parts was assembled with use of specific interface joints.

To design the proposed structure of the minimum weight, the algorithm of optimization of the wing box with different structural layouts has been developed.

The basic objectives were sizing of load-bearing elements for each of the layouts, and to search for the interface position, providing minimum weight of the wing box structure. These tasks were solved with use of genetic algorithm. Additional task was connected with matching of different structural layouts in adjacent sections. The results of parametric studies of wing box structure was used to solve this task.

The validation researches basing on weight analysis for the wing box of regional aircraft have shown good potential of the proposed hybrid layout for the weight reduction. The rational variant of the length of the wing box section with lattice-truss structural layout was $65-73 \%$ of the wing box. This variant meets the constraints, and has the weight benefit (13$17 \%$ ) compared with conventional structural layout.

This work was supported by the Subsidy of Ministry of Education and Science of the Russian Federation, Agreement \#14.628.21.0009, project identification number RFMEFI628180009.

\section{References}

1. E. Dubovikov, V. Fomin, I. Kondakov, A. Shanygin, Development of rational hybrid fuselage structure for prospective civil aircraft // 7th EASN Conf. Aerostr., Warshava, Poland, (2017)

2. A. Chernov, D. Fomin, V. Grishin, I. Kacharava, A. Shanygin, Development of Lightweight and Reliable Joints for Airframes Based on Unidirectional Composite Elements// Proc. of $30^{\text {th }}$ ICAS Conf., Daejeon, Korea, (2016)

3. E. Dubovikov, V. Fomin, I. Mareskin, A. Shanygin, M. Zichenkov, Designing Procomposite Truss Layout for Loadbearing Aircraft Structures // Fatigue Fract. Eng. Mater. Struct. (FFEMS), 40(10) : 1612-1623, (2017)

4. A. Mallika, Discrete Optimization of Truss Structure Using Genetic Algorithm // International Journal of Recent Development in Engineering and Technology, 3(1) : 105-111, (2014)

5. S. Osman, E. Atef, S. Tharwat, H. Osman, Optimization of Plane and Space Trusses Using Genetic Algorithms // International Journal of Engineering and Innovative Technology (IJEIT), 3(7) : 66-73, (2014) 